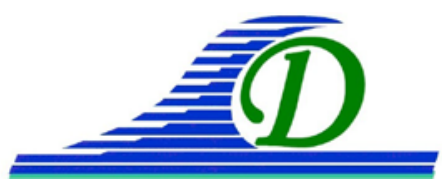

XIII İ̀es Journées Nationales Génie Côtier - Génie Civil Dunkerque, 2-4 juillet 2014

DOI:10.5150/jngcgc.2014.046 C Editions Paralia CFL

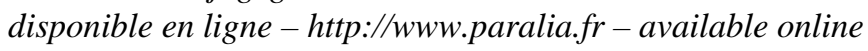

\title{
Quantification du transport sédimentaire éolien réel du littoral de Saidia - Cap de l'Eau (Maroc nord-est)
}

\author{
Hicham LASGAA ${ }^{1}$, Abdelkader SBAI ${ }^{1}$, Taieb BOUMEAZA ${ }^{1}$
}

1 Département de Géographie. Université Hassan II, Mohammedia-Casablanca, Maroc. hichamlasgaa@yahoo.fr

\section{Résumé :}

Situé à l'extrême nord-est du Maroc, le littoral de Saidia-Cap de l'Eau est constitué d'une plage sableuse qui s'étend sur $20 \mathrm{~km}$. La déflation éolienne est l'un des problèmes affectant cette côte. Cela se manifeste clairement dans le transport des sables jusqu'aux terrains situés en arrière dune et dans l'accumulation des sables au bord des constructions récemment aménagées. Des mesures instantanées ont été effectuées depuis le haut de la dune jusqu'à la basse plage sur six profils en utilisant les pièges à sable de type LEATHERMAN (1978) pour quantifier le transport sédimentaire éolien in situ. Pendant les campagnes de mesures sur le terrain, le vent a été mesuré par une station anémométrique mobile. Les résultats de cette étude montrent que $44,4 \mathrm{~kg} / \mathrm{m} / \mathrm{h}$ en moyenne des sables piégés sur le terrain (24 pièges en total) sont associés à des vents dont la vitesse moyenne est de $7 \mathrm{~m} / \mathrm{s}$ environ mesuré à $2 \mathrm{~m}$ d'altitude. Le transit maximum mesuré est au niveau du bas et du milieu de la plage, tandis que le transport le plus réduit est piégé dans les sites les plus proches de la ligne de rivage instantanée du fait de l'influence de l'humidité du substrat qui empêche les sédiments de se déplacer facilement et en haut de la dune où la végétation capte les sables en transit. L'analyse granulométrique des sédiments piégés montre une simple diminution de la taille des grains des sédiments de la basse plage vers le haut de la dune.

Mots clés : Transport éolien, Piège à sable, Vents, Sédiment, Dune, Plage, Saidia-Cap de l'Eau, Maroc nord-est.

\section{Introduction}

L'étude du transport sédimentaire éolien constitue un sujet fondamental dans la morphodynamique des littoraux sableux, notamment la formation et la mobilité des dunes bordières. Le vent constitue un agent principal du transport éolien. Sa vitesse, sa direction et sa durée sont des agents essentiels qui doivent être fondamentalement étudiés. La capacité du vent à transporter les sédiments varie en fonction des caractéristiques locales. A cet égard, les particules les plus fines sont transportées en suspension et en saltation, tandis que les grossières sont transportées en roulement. La pente, la gravité et le couvert végétal sont aussi des facteurs modifiants la capacité du transport par le vent. L'adhérence, cependant faible, que donne l’humidité aux grains 
d'un sable mouillé suffit à arrêter leur mise en mouvement jusqu'à ce qu'ils soient secs (BAGNOLD, 1941).

Il existe plusieurs modèles pour quantifier le transport réel à différentes altitudes le long des plages (BSNE, POLCA, MWAC, SUSTRA, saltiphone, Sartorius, Piège-Owens, Piège -Plateau, Piège de Leatherman 1978). Le principe commun entre ces nombreux appareils est d'assurer le piégeage des particules transportées au sein de collecteurs et de laisser le vent sortir. Parmi eux, les pièges de type LEATHERMAN (1978) ont été utilisés pour quantifier le transport éolien in situ sur le littoral de Saidia - Cap de l'Eau. Ils sont simples et faciles à fabriquer et à déplacer sur le terrain.

\section{Présentation de la zone d'étude}

La façade maritime qui fait l'objet de cette étude s'étend sur $20 \mathrm{~km}$ de Oued Kiss à l'Est jusqu'à Cap de l’Eau à l'ouest (figure 1). La morphologie générale de cette côte est très peu variée. Au milieu de la plaine côtière de Saïdia - Cap de l’Eau, l'Oued Moulouya divise la côte en deux parties. Ce dernier marque la source essentielle des sédiments qu'on trouve sur la plage. La plage est formée par des sables fins et moyens qui forment une très belle plage. L'arrière plage de cette zone est formée par des dunes sableuses de direction NNW-SSE constituant ainsi une protection naturelle des terres basses en arrière et servant à l'alimentation de la plage en période du mauvais temps. Deux types de dunes sont distingues : les nebkas littorales (petites dune asymétrique formée par la présence d'un obstacle végétal) et les dunes parallèles au littoral : cordons dunaires correspondant à d'anciennes dunes bordières marquant l'avancée du trait de côte. Le largueur de la plage est souvent plus de $100 \mathrm{~m}$ et d'une pente faible. Les dunes sont caractérisées par des topographies basses ( 0 à $6 \mathrm{~m}$ ), une urbanisation intense et un intérêt écologique, touristique et économique important (SBAI \& LASGAA, 2012).

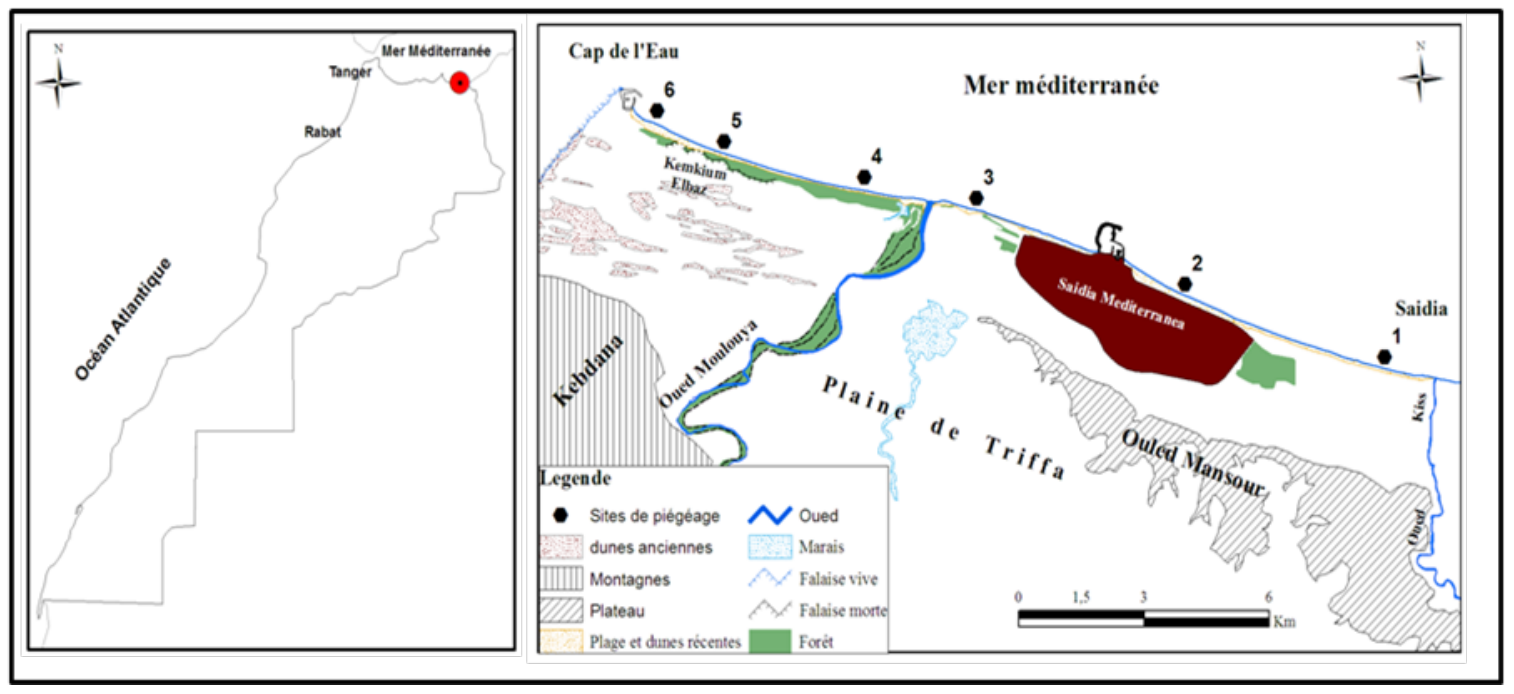

Figure. 1. Situation de la zone d'étude. 


\section{XIII ${ }^{\text {èmes }}$ Journées Nationales Génie Côtier - Génie Civil \\ Dunkerque, 2-4 juillet 2014}

\section{Méthode de quantification du transport sédimentaire éolien}

Une large gamme des modèles des pièges à sables pour collecter les sédiments est actuellement en cours d'utilisation par les chercheurs dans le monde. Parmi eux, les pièges à sables de type LEATHERMAN (1978) sont couramment utilisés par les chercheurs dans le monde (SHERMAN et al., 1998 ; SABATIER, 2001 ; MEURFEREC \& RUZ, 2002). Ils sont simples, faciles à fabriquer et peu coûteux. Les pièges sont constitués par des cylindres PVC de $10 \mathrm{~cm}$ de diamètre et $75 \mathrm{~cm}$ de haut, enfoncés verticalement dans le sable jusqu'à $25 \mathrm{~cm}$ (figure 2). Le cylindre comporte deux ouvertures : une ouverture de $1 \mathrm{~cm}$ face au vent et une ouverture de $2.5 \mathrm{~cm}$ sous le vent, équipé d'un maillage $0,063 \mathrm{~mm}$ pour laisser passer le vent et permettre le piégeage des sédiments.

Quatre pièges ont été déployés simultanément pour mesurer le transport sédimentaire au niveau du front de la plage, du milieu de la plage, du pied de dune et sur la dune bordière (figure 2). Nous avons pris six (6) profils d'échantillonnage répartis sur le long du littoral de Saidia - Cap de l'Eau (figure 1). Le sable piégé a été ensuite séché, pesé et analysé au laboratoire de géomorphologie pour déterminer les caractéristiques granulométriques des sédiments.

Pour mesurer les conditions météorologiques associées transport éolien, nous avons installé une station météorologique numérique professionnelle de marque OregonScientific dans chaque secteur de piégeage. La hauteur de l'anémomètre est de $2 \mathrm{~m}$ installé au milieu de la plage. Les mesures de la vitesse du vent ainsi que leur direction sont transmises toute les 14 secondes sur un écran sans fil. A la fin de chaque période de mesure, les données météorologiques (vitesse et direction du vent, humidité de l'air, température...) sont récupérées et transmises sur ordinateur à l'aide du logiciel Weather OS 4.

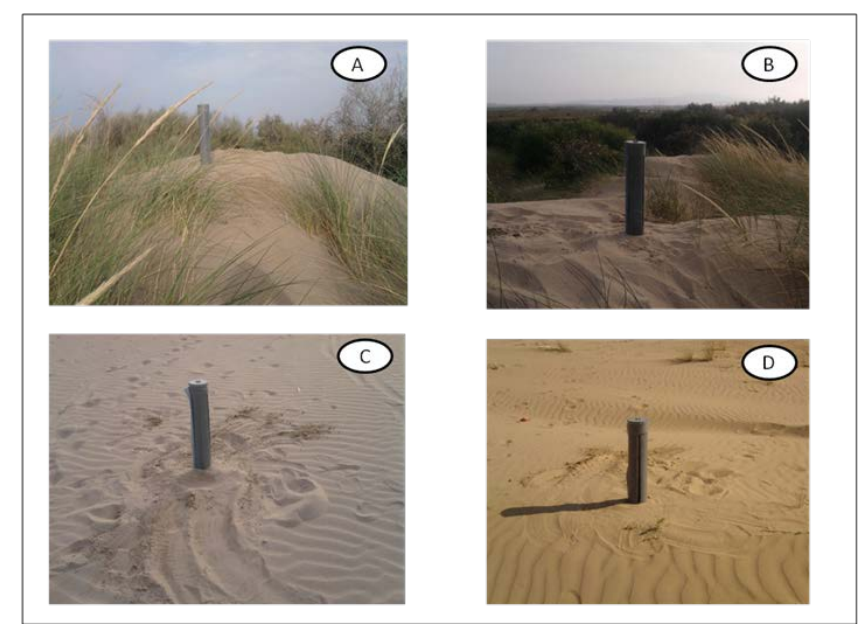

Figure 2. Pièges à sable de type LEATHERMAN (1978). A : piège déployé au niveau du haut de la dune. $B$ : au niveau du pied de la dune. $C$ : en haut de la plage.

$D$ : au niveau de la basse plage. 


\section{Thème 2 - Dynamique sédimentaire}

\section{Résultats et discussion}

4.1 Conditions de forçages pendant les campagnes de mesures

La vitesse et la direction du vent ont été mesurées in-situ pendant la durée de piégeage (30 min). Nous avons effectué trois campagnes de terrain pour quantifier le transport sédimentaire réel. La première était le 06/04/2013 et concernait les sites 5 et 6 . La deuxième sortie de terrain était le 07/04/2013 dans les sites 3 et 4 . La troisième campagne de mesure effectuée dans les sites 1 et 2 a eu lieu le 13/04/2013 (figure 1). Les résultats des conditions de piégeage sont reportés dans le tableau 1.

Le vent est insuffisamment fort pour générer un transport sédimentaire considérable pendant la première campagne de mesure. La vitesse moyenne est de l'ordre de 4 à 5.5 $\mathrm{m} / \mathrm{s}$ dans les sites 5 et 6 . Pendant la $2^{\text {eme }}$ campagne de mesure, la vitesse du vent est modérément forte, elle varie de 8,5 à $5 \mathrm{~m} / \mathrm{s}$ en moyenne. Pendant la $3^{\text {ème }}$ sortie de terrain, la vitesse du vent mesurée est suffisamment forte pour générer un transport sédimentaire éolien remarquable. Sa vitesse varie de 5,5 à $8 \mathrm{~m} / \mathrm{s}$ au niveau du site 1 et 8 à $8,6 \mathrm{~m} / \mathrm{s}$ dans le site 2 .

Nous avons mesuré simultanément la température, l'humidité de l'air et la pression atmosphérique. Cependant, nous constatons que les vents forts s'accompagnent d'une diminution de la pression atmosphérique. Cette dernière était de 1010 hPa. L'humidité de l'air varie respectivement de $46 \%$ à $40 \%$.

Les sites de mesure 1, 3, 4, 5 et 6 se caractérisent par une largeur de plage de plus de $100 \mathrm{~m}$ environ, tandis que le site 2 présente une largeur de l'ordre de $20 \mathrm{~m}$ à $30 \mathrm{~m}$.

La pente de la plage mesurée pendant chaque compagnie de mesure est souvent faible. Elle varie de 1,6\% à $4 \%$ sur les sites de piégeages 1, 3, 4, 5 et 6 et d'une pente forte de $10 \%$ au niveau du site 2 .

Tableau 1. Condition de piégeages.

\begin{tabular}{llll}
\hline Site & Vitesse du vent en $\mathrm{m} / \mathrm{s}$ & Largueur de la plage & la Pente en\% \\
\hline 1 & 7,5 & 120 & 1,6 \\
2 & 8,3 & 20 & 10 \\
3 & 8,5 & 100 & 4,0 \\
4 & 5,0 & 140 & 2,8 \\
5 & 5,5 & 120 & 1,6 \\
6 & 6,4 & 110 & 1,8 \\
\hline
\end{tabular}

\subsection{Transport sédimentaire total réel}

Vingt piégeages ont été effectués sur six profils lors de trois campagnes de mesures sur le terrain. La quantité totale des sables piégés est de $0,74 \mathrm{~kg} / \mathrm{m} / \mathrm{min}$, soit $44,4 \mathrm{~kg} / \mathrm{m} / \mathrm{h}$ pour l'ensemble des collecteurs. Le transport éolien maximum au niveau de la limite du SIBE de la Moulouya avec la station balnéaire "Saidia Méditerranéa" (site 2), (figure 3). 


\section{XIII ${ }^{\text {èmes }}$ Journées Nationales Génie Côtier - Génie Civil \\ Dunkerque, 2-4 juillet 2014}

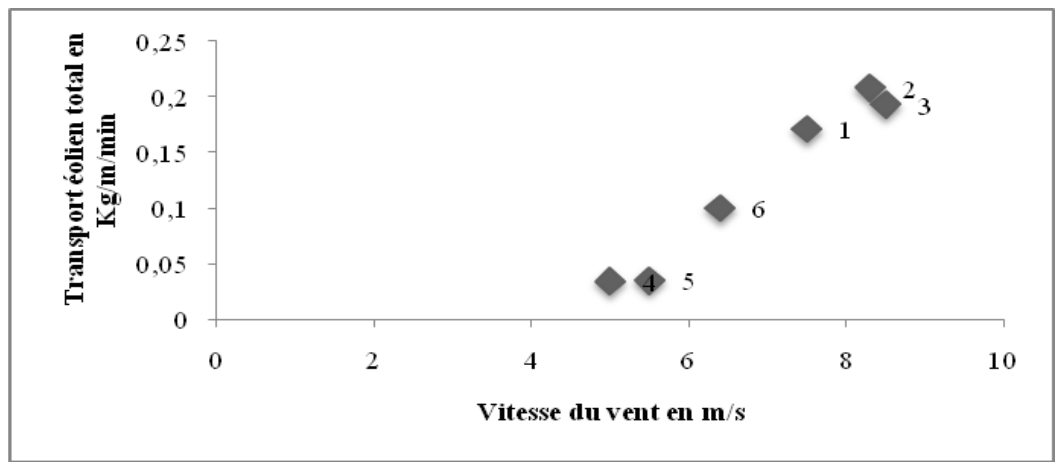

Figure 3. Transport réel total.

Le taux du transport éolien total mesuré au niveau du site 1 et 2 durant la première campagne de mesure est faible, il varie de 0,034 à $0,035 \mathrm{~kg} / \mathrm{m} / \mathrm{min}$ (soit 2,04 à $2,1 \mathrm{~kg} / \mathrm{m} / \mathrm{h}$ ). Cette valeur et est en relation avec un vent de $5,5 \mathrm{~m} / \mathrm{s}$ de direction NO. Durant la période de mesure sur le terrain, le taux du transport total calculé est de 0,034 à $0,2 \mathrm{~kg} / \mathrm{m} / \mathrm{min}$ (soit 2,04 à $12 \mathrm{~kg} / \mathrm{m} / \mathrm{h}$ ) en moyenne associé à des vents de $5 \mathrm{~m} / \mathrm{s}$ au niveau du site 4 et de $8.3 \mathrm{~m} / \mathrm{s}$ au niveau du site 2. Le taux du transport éolien est maximum au niveau des sites 1,2 et 3 ; il est associé à un vent de 7,5 à $8,5 \mathrm{~m} / \mathrm{s}$ de direction NO. Le taux du transport éolien le plus élevé est mesuré sur les points les plus hauts de la plage, où la vitesse du vent est plus forte et le sable est sec et plus disponible au transport.

Le taux du transport le plus faible se trouve dans les points les plus proches de la mer (niveau des points d) où l'humidité des sables empêche les particules de sables de se déplacer. Par ailleurs, les plantes des dunes empêchent les sédiments de se déplacer, constituant ainsi un obstacle biologique de fixation des dunes.

Ceci, montre d'une manière primordiale le rôle de l'intensité et de la vitesse des vents dans le transport des sédiments. L'augmentation de la vitesse du vent s'accompagne d'une augmentation du taux du transport sédimentaire total.

\subsection{Organisation latérale du transport sédimentaire éolien}

La quantité des sédiments transportés par le vent varie d'un site à l'autre suite à la variation de l'intensité des vents soufflant pendant la période de mesure et la variabilité des unités morphologique des profils. La figure 4 illustre les résultats des expériences de terrain en pourcentage : la part des sables collectés sur chaque point de mesure par rapport à la somme totale de l'ensemble des quantités piégées sur le même profil.

Sur le haut de la dune (a), le taux du transport sédimentaire total capturé est le plus réduit, il varie de 5,84 à 14,7\% du transport total. Ce point de mesure est souvent fixé par un couvert végétal moyennement dense. Au niveau du pied de la dune (b), le transport éolien total varie de 10 à 15,5\% du transport total au niveau des sites 1, 2, 3 et 6 et de 23,52 à 25,71\% environ au niveau des sites 4 et 5 . Sur le $3^{\text {ème }}$ point de piégeage 


\section{Thème 2 - Dynamique sédimentaire}

(c), les sédiments transportés représentent environ 10 à 31,42\% du transport éolien total. Cette zone de mesure est presque dégagée et dépourvue du couvert végétal. Au milieu de la plage (d), le transport sédimentaire par le vent représente 28,5 à 71\% du transport total.

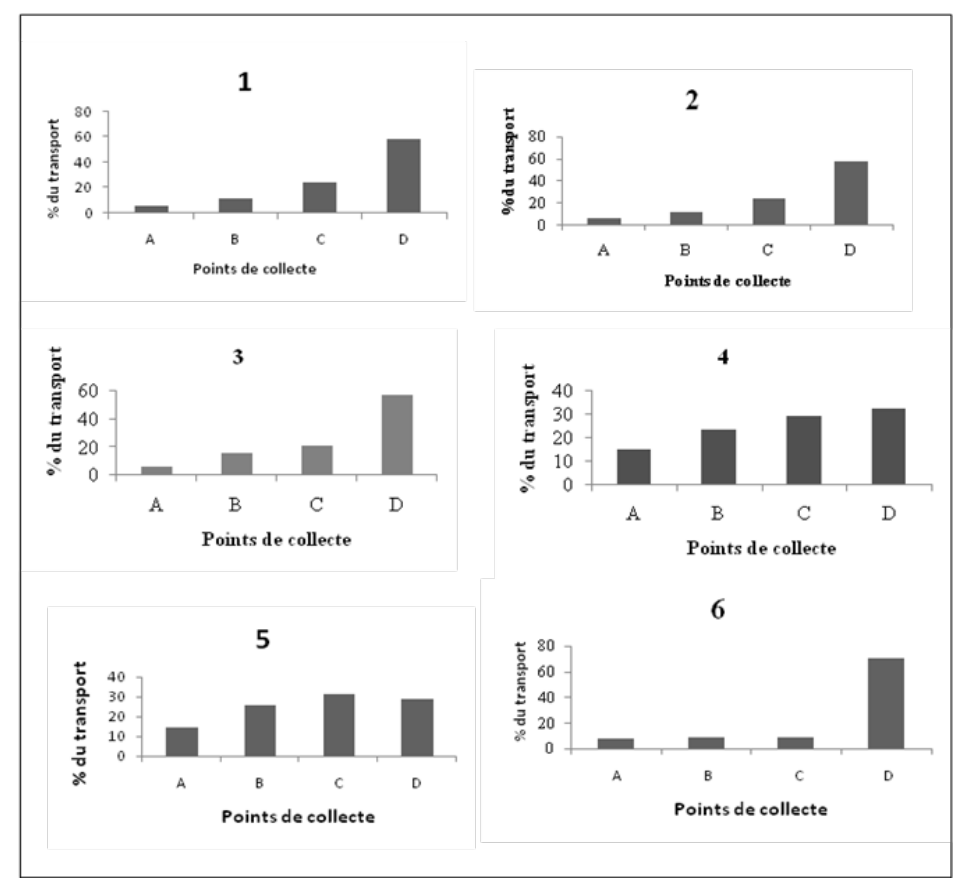

Figure 4. Distribution latérale du transport éolien. 1, 2, 3, 4, 5 et 6 représentent les sites d'expérimentation. $A, B, C$ et $D$ représentent les points de collecte.

Sur tous les profils, on note un gradient spatial de la quantité des sables transportés du bas de la plage vers le haut de la dune. Ce gradient nous permet de mettre en évidence que les dunes sont des zones de dépôts puisque elles correspondent à des zones de diminution du transport éolien. Par ailleurs, le bas et le milieu de la plage constituent une zone source pour les sédiments, c'est sur cette zone que le transport sédimentaire éolien est le plus élevé.

Généralement, le transport sédimentaire éolien vertical dans la partie subaérienne de la plage est en relation directe avec deux paramètres principaux : la vitesse du vent et la topographie de la plage. Le transport maximum est situé au milieu de la plage. Par ailleurs, le transport le plus réduit est enregistré en sommet des dunes couvertes par la végétation (a). Les conditions anémométriques influencent la quantité des sédiments mobilisés. Les vents les plus forts correspondent à un transport éolien maximal.

\subsection{Caractéristiques granulométriques des sédiments transportés par le vent}

La représentation graphique des résultats de l'étude granulométrique de 23 échantillons collectés dans la zone subaérienne fait apparaitre une faible diminution de la taille des 


\section{XIII ${ }^{\text {èmes }}$ Journées Nationales Génie Côtier - Génie Civil \\ Dunkerque, 2-4 juillet 2014}

grains des sédiments du bas de plage vers le haut de la dune (figure 5). Plusieurs matériaux sédimentaires ont été collectés pendant les campagnes de terrain; nous distinguons, dans ce cadre, des matériaux organiques (fragments des plantes ...), les fragments de coquillage.

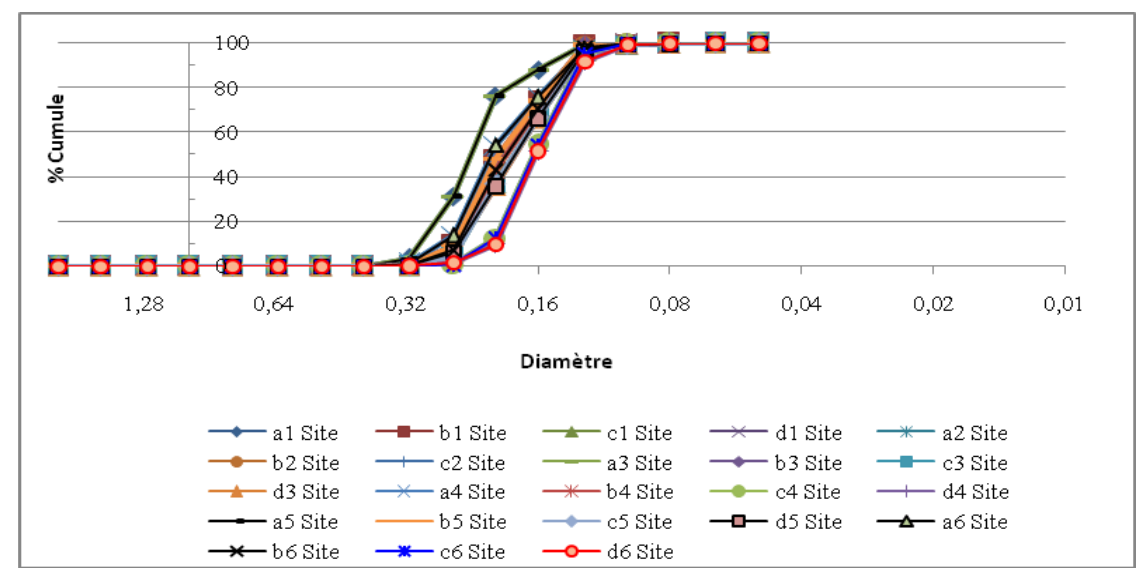

Figure 5. Courbes granulométriques des échantillons piégés.

Au niveau du bas de la plage, le diamètre moyen des sédiments $\left(D_{50}\right)$ est compris entre 0,19 et $0,22 \mathrm{~mm}$. La fraction sableuse la plus grossière a été trouvée au niveau des sites 3 et 4 . Les sédiments les plus fins correspondent aux sites 2, 5 et 6. Généralement, la fraction sableuse la plus grossière est située près du rivage et la plus fine se trouve au sommet de la dune.

Au milieu de la plage $(\mathrm{c})$, le diamètre moyen $\left(D_{50}\right)$ correspond à un sédiment moyen de taille comprise entre 0,22 et $0,80 \mathrm{~mm}$. La fraction la plus grossière est collectée au niveau de la rive gauche et droite de la Moulouya (Sites 3 et 4), alors que la plus fine se trouve au niveau de la falaise de Kemkoume El Baz (Site 5).

Au niveau du pied de dune (b), les résultats de l'analyse granulométrie semblent similaires aux points de mesures précédents. Cependant, la taille des sédiments échantillonnés varie de 0,17 à $0,20 \mathrm{~mm}$. L'évolution des courbes présente une prépondérance vers des valeurs plus fines vers le sommet des dunes à travers l'ensemble des échantillons analysés.

Au niveau du haut de la dune (a), le diamètre des grains présente les valeurs les plus fines de touts les profils étudiés. La taille moyenne de la fraction sableuse varie de 0,17 à $0,19 \mathrm{~mm}$.

Spatialement, nous constatons une diminution de la taille des sables collectés sur chaque profils de mesure de la zone intertidale vers le sommet de la dune d'une part et de l'embouchure de la Moulouya vers Saidia et vers Cap de l'Eau. Ceci correspond à une zone source principale des sédiments que l'on trouve sur la plage à savoir l'oued Moulouya et son bassin versant. 


\section{Thème 2 - Dynamique sédimentaire}

\section{Conclusion}

Le transport sédimentaire éolien a été quantifié en utilisant des pièges à sable conventionnel de type LEATHERMAN (1978) dans des conditions de pente relativement différentes. Généralement, $97,8 \mathrm{~kg} / \mathrm{m} / \mathrm{h}$ en moyenne piégés sur le terrain sont associés à des vents de $7 \mathrm{~m} / \mathrm{s}$ environ.

Un gradient spatial de la quantité des sables collectés est observé sur les mêmes profils du bas de la plage vers le sommet des dunes accompagné souvent par une diminution du diamètre des sables de la zone intertidale vers les cordons dunaires.

Cette étude montre que les variations spatiales du transport sédimentaire éolien peuvent être différentes à petite échelle et sur le même secteur et sous les même conditions. Cependant, plusieurs facteurs peuvent entrainer cette variabilité, comme cela a été démontré dans de nombreuses études : au niveau de la ligne de rivage instantanée, la marée haute et le va-et-vient de la mer influence l'humidité du substrat limite considérablement le transport éolien. Au niveau des dunes, le tapis végétal capte les sables transportés par le vent, constituant ainsi une zone de dépôt et d'accumulation des sédiments.

En conclusion, la vitesse, la direction et la durée d'action du vent, la nature de la surface d'envol ou de déflation, la taille des particules des sédiments et la teneur en humidité des sédiments, sont autant de facteurs primordiaux dans le transport sédimentaire éolien.

\section{Références bibliographiques}

BAGNOLD R.A. (1941). The physics of wind blown sands and desert dunes. Methuen, London, 265 p.

LEATHERMAN S.P. (1978). A new Aeolian sand trap design. Sedimentology, Vol. 25, pp 303-306. http://dx.doi.org/10.1111/j.1365-3091.1978.tb00315.x

MEUR-FEREC C., RUZ M.-H. (2002). Transports éoliens réels et théoriques en haut de plage et sommet de dune (Wissant, Pas-de-Calais, France). Géomorphologie : relief, processus, environnement. octobre-décembre, Vol. 8, n ${ }^{4}$. pp 321-334.

SABATIER F. (2001). Fonctionnement et dynamiques morpho-sédimentaires du littoral du delta du Rhône. Thèse de doctorat. Université de Droit, d’Economie et des Sciences d’Aix-Marseille III. Faculté des Sciences et Techniques de Saint Jérôme. 285 p.

SBAI A., LASGAA H. (2012). Vulnérabilité et impacts des risques associés à l'élévation du niveau de la mer dans la plaine littorale de Saidia - Cap de l'Eau (Maroc nord-est). XII ${ }^{\text {èmes }}$ Journées Nationales Génie Côtier - Génie Civil, Cherbourg, pp 419-426. http://dx.doi.org/10.5150/jngcgc.2012.045-S

SHERMAN D.J., JACKSON D.W.T., NAMIKAS S.L., WANG J. (1998). Wind-blown sand on beaches: an evaluation of models. Geomorphology, Vol. 22, pp 113-133. http://dx.doi.org/10.1016/S0169-555X(97)00062-7 